\title{
DESEMPENHO PROFISSIONAL OU DOMÉSTICO DAS PACIENTES EM QUIMIOTERAPIA PARA CÂNCER DE MAMA
}

Lourdes Conceição Martins*1, Celso Ferreira Filho ${ }^{2}$, Auro Del Giglio33, Daniela Armonia Munhoes ${ }^{1}$, Ligia Lopes Balsalobre Trevizan ${ }^{1}$, Livia Grigoriltchuk Herbst ${ }^{1}$, Mariana da Cunha Viera ${ }^{1}$, Patrícia Taranto ${ }^{1}$, Susy Cunha Pachon ${ }^{1}$

Trabalho realizado na Faculdade de Medicina do ABC, Santo André, SP

*Correspondência:

R. Abraham Bloemaert,

126

d. das Vertentes.

São Paulo - SP.

CEP 05541-320

\begin{abstract}
RESUMO
Овјетіvos. Analisar os aspectos epidemiológicos, clínicos, desempenho profissional e doméstico nas pacientes com câncer de mama em tratamento quimioterápico.

Métodos. 0 estudo foi transversal, com 52 mulheres entrevistadas, divididas em dois grupos: afastadas e não afastadas das atividades profissionais e domésticas, durante 0 tratamento com quimioterapia para câncer de mama. As pacientes foram provenientes de dois hospitais, um situado no município de Santo André e o outro no município de São Bernardo do Campo. Ambos são hospitais públicos de referência, que atendem a população de Santo André, São Bernardo do Campo, São Caetano do Sul, Diadema e Mauá, que são municípios vizinhos, pertencentes ao Estado de São Paulo, próximos da capital. Utilizou-se o questionário WPAI-GH (Work Productitity and Actitity Impairment) para avaliar a atividade profissional e/ou doméstica das pacientes.

Resultados. As pacientes tinham idade média de 55,7 anos ( $d p=13,8$ ), a maior parte de etnia branca $(88,5 \%)$, casada $(55,8 \%)$, assalariada $(65,3 \%)$ e a maioria se afastou das atividades $(51,0 \%)$, com estadiamento da doença mais avançado $(p<0,05)$, fadiga e náuseas $(p<0,05)$. A média do WPAI GH foi $67,04(\mathrm{dp}=5,62)$ para as pacientes afastadas, e 49,17 (dp =6,89) para as não afastadas do trabalho (teste $U$ de Mann-Whitney: $p=0,04$ ).

Conclusão. A quimioterapia acarreta diminuição da capacidade produtiva em parte significativa das mulheres com câncer de mama. 0 estadiamento mais avançado da neoplasia associou-se positivamente com o afastamento das atividades diárias das pacientes, provavelmente decorrente dos efeitos colaterais provocados, como fadiga e náuseas.
\end{abstract}

Unitermos: Neoplasia da mama. Quimioterapia. Avaliação de desempenho.

\section{InTRODUÇÃO}

0 câncer é uma doença de grande incidência no Brasil. No ano de 2006 foram relatados 472.050 novos casos entre homens e mulheres a cada 1.000 .000 de habitantes ${ }^{1}$.

0 câncer de mama é o maior responsável pelos óbitos decorrentes de neoplasias em mulheres no Brasil. Segundo o Instituto Nacional do Câncer (INCA), a estimativa foi de 48.930 casos no ano de $2006^{1}$.

Os tratamentos utilizados em neoplasias têm como objetivos a cura, evitar a recidiva, e aumentar a sobrevida com qualidade para o paciente. Durante anos, a cirurgia foi considerada a única opção para os casos de câncer. Com a evolução das pesquisas, novos tratamentos como a radioterapia, hormonioterapia, imunoterapia e a quimioterapia foram desenvolvidos. Apesar de serem cada vez mais específicos, ainda provocam muitos efeitos colaterais que comprometem a saúde e a qualidade de vida dos pacientes ${ }^{2,3,4}$.
$\mathrm{Na}$ última década a sobrevida de pacientes com câncer de mama aumentou com o diagnóstico precoce e utilização da quimioterapia adjuvante. A complexidade dos tratamentos quimioterápicos e sua duração tornam necessária a melhor compreensão do desempenho profissional e doméstico das pacientes neste período ${ }^{5}$.

Os sintomas decorrentes mais relevantes do tratamento são: depressão, ansiedade, insônia, fadiga, stress psicológico, vômi-

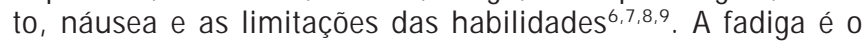
sintoma mais debilitante, aumentando o tempo de retorno ao trabalho, enquanto a ansiedade e a depressão ocasionam 0 afastamento das atividades normais ${ }^{10}$.

As pacientes em quimioterapia com atividade remunerada apresentaram limitações físicas e elevado percentual de faltas no trabalho devido à realização de exames complementares e continuidade no tratamento ${ }^{11}$.

Entretanto, a permanência no trabalho auxilia a recuperação do tratamento, aumentando a autoestima, controle emocional,

1. Professora da disciplina de Saúde Coletiva da Faculdade de Medicina do ABC, Santo André, SP

2. Professor titular da disciplina de Propedêutica da Faculdade de Medicina de Santo Amaro. Professor da disciplina de Cardiologia da FMABC, Santo André, SP

3. Professor titular da disciplina de Oncologia da FMABC, Santo André, SP 
distração e redução da ansiedade, resultando em maior valorização da vida, aceitação e compromisso com o tratamento, influindo positivamente no processo de cura ${ }^{12}$.

Os gastos hospitalares associados ao elevado absenteísmo no trabalho, decorrente da recuperação das pacientes em tratamento, determinam diminuição da produtividade pessoal e grande impacto social ${ }^{13}$.

O presente estudo analisou os aspectos epidemiológicos, clínicos, desempenho profissional e doméstico em um grupo de pacientes com câncer de mama em tratamento quimioterápico.

\section{Métodos}

Este é um estudo transversal, com entrevista de pacientes provenientes de dois hospitais, um situado no município de Santo André e o outro no município de São Bernardo do Campo, durante o tratamento quimioterápico para câncer de mama.

Ambos são hospitais públicos de referência, que atendem a população de Santo André, São Bernardo do Campo, São Caetano do Sul, Diadema e Mauá, que são municípios vizinhos, pertencentes ao Estado de São Paulo, próximos da capital.

Foram incluídas todas as mulheres com idade mínima de 21 anos, com diagnóstico de câncer de mama em tratamento com quimioterapia durante o primeiro semestre de 2007, que assinaram o termo de consentimento livre e esclarecido, e, portanto, concordaram em participar do estudo. 0 estudo foi aprovado pelo Comitê de Ética em Pesquisa da Faculdade de Medicina do $A B C$.

Dois questionários foram utilizados: um com informações sobre escolaridade, moradia, estado civil, estadiamento do câncer, doenças preexistentes e sintomas, e outro: WPAI-GH (Work Productivity and Activity Impairment - Genera Health). U ma versão brasileira foi confeccionada e validada no Brasil14.

O questionário WPAI - GH mede os efeitos da saúde (sintomas gerais e específicos) sobre a produtividade no trabaIho e fora do trabalho. Tem sido o instrumento mais frequentemente aplicado e analisado para diversas doenças. Este questionário detecta os dias de absenteísmo (faltas em horas de trabalho devido a um problema de saúde) e de presenteismo (redução da eficácia de uma pessoa no trabalho devido a um problema de saúde). A perda da produtividade é entendida, portanto, neste questionário, como a diminuição da capacidade total de trabalho, mensurada pela soma do absenteísmo e do presenteismo.

O WPAI - GH é composto por seis questões que pedem ao participante informações: 1- se estão empregados atualmente ou não; 2- sobre o número de horas não trabalhadas devido a problemas de saúde; 3- sobre o número de horas não trabalhadas por outras razões (por exemplo, férias); 4 - sobre o número de horas realmente trabalhadas; 5- quanto os problemas de saúde afetam sua produtividade quando estava trabal hando e 6 quanto os problemas de saúde afetaram suas atividades diárias nos últimos sete dias.

As duas últimas perguntas são avaliadas em uma escala de 10 pontos, variando de 0 (nenhum efeito no trabalho) a 10 (os problemas de saúde impediram que a pessoa trabalhasse).

\begin{tabular}{|c|c|c|c|c|}
\hline \multirow[b]{4}{*}{ Etnia } & \multirow{3}{*}{$\begin{array}{l}\text { Total } \\
\mathbf{N}^{\circ}(\%)\end{array}$} & \multicolumn{2}{|c|}{ Afastamento das atividades } & \multirow[t]{3}{*}{$\mathbf{p}^{*}$} \\
\hline & & Sim & Não & \\
\hline & & \multicolumn{2}{|c|}{$\mathbf{N}^{\circ}(\%)$} & \\
\hline & & & & \\
\hline Branca & $46(88,5)$ & $22(78,6)$ & $24(100,0) *$ & 0,016 \\
\hline Negra & $6(11,5)$ & $6(21,4)$ & 0 & \\
\hline \multicolumn{5}{|l|}{ Estado Civil } \\
\hline Solteira & $10(19,2)$ & $4(14,3)$ & $6(26,1)$ & \\
\hline Casada & $29(55,8)$ & $16(57,1)$ & $13(56,5)$ & 0,41 \\
\hline Separada & $6(11,5)$ & $3(10,7)$ & $3(13,0)$ & \\
\hline Viúva & $6(11,5)$ & $5(17,9)$ & $1(4,4)$ & \\
\hline \multicolumn{5}{|l|}{ Escolaridade } \\
\hline Analfabeto & $9(17,3)$ & $5(17,9)$ & $4(17,4)$ & 0,38 \\
\hline Fundamental incompleto & $17(32,7)$ & $8(28,6)$ & $9(39,1)$ & \\
\hline Fundamental completo & $9(17,3)$ & $3(10,7)$ & $6(26,1)$ & \\
\hline Médio incompleto & $2(3,8)$ & $2(7,1)$ & 0 & \\
\hline Médio completo & $7(13,5)$ & $5(17,9)$ & $2(8,7)$ & \\
\hline Superior & $7(13,5)$ & $5(17,9)$ & $2(8,7)$ & \\
\hline \multicolumn{5}{|l|}{ Profissão } \\
\hline Assalariada & $32(65,3)$ & $19(76,0)$ & $13(54,2)$ & 0,10 \\
\hline Do lar & $17(34,7)$ & $6(24,0)$ & $11(45,8)$ & \\
\hline
\end{tabular}

As questões são computadas de acordo com regras de cálculo específicas, gerando escores. Altos escores indicam licença-saúde prolongada ou produtividade diminuída.

Este questionário é considerado um importante instrumento, confiável e útil para a mensuração do impacto de problemas de saúde sobre a produtividade.

Foram considerados dois grupos, pacientes afastadas e não afastadas das atividades profissionais e domésticas.

Após a obtenção dos dados, foi feita a análise descritiva de todas as variáveis do estudo. As variáveis qualitativas foram apresentadas em valores absolutos e relativos. As variáveis quantitativas foram apresentadas em termos de seus valores de tendência central e dispersão $0^{15}$.

Para verificar a associação entre as variáveis qualitativas, foi utilizado o teste de Qui quadrado ${ }^{16}$. Para se comparar duas proporções foi utilizado o teste de comparação entre duas proporções ${ }^{16}$. Para a comparação da idade e do domínio do questionário WPAI, entre as pacientes que se afastaram ou não do trabalho, foi utilizado o teste $U$ de Mann-Whitney, pois esta variável não apresentava aderência à curva normal (teste de Kolmogorov-smirnov) e homogeneidade das variâncias (teste de Levene) ${ }^{17}$, com $5 \%$ de nível de significância. 0 pacote estatístico utilizado foi o SPSS 14.0 for windows.

\section{Resultados}

Responderam aos questionários 52 pacientes, de dois hospitais públicos de referência para a doença, da região do $A B C$.

$\mathrm{Na}$ Tabela 1 são apresentados os dados biodemográficos para o grupo todo e estratificado por afastamento das 
atividades. As pacientes tinham idade média de 55,7 anos $(d p=13,8)$, a maior parte de etnia branca $(88,5 \%)$, casada $(55,8 \%)$, assalariada $(65,3 \%)$, e proporção de escolaridade com distribuição equivalente. Os dados biodemográficos estudados não tiveram associação estatística (teste de Qui quadrado, $p>0,05)$ com 0 afastamento do trabalho das pacientes, exceto para a etnia. Para cada variável, a distribuição entre o afastamento e não afastamento do trabalho tiveram distribuição equivalente.

$\mathrm{Na}$ Tabela 2 são apresentados os seguintes dados clínicos da população estudada: menopausa, reposição hormonal, cirurgia prévia da mama, hipertensão arterial, Diabetes mellitus, dislipidemia, disfunção da tireóide, doença cardiovascular e estadiamento da neoplasia (I - II e III - IV). A maior parte das pacientes estava no período de menopausa (Teste de comparação entre duas proporções: $p<0,05)$, sem reposição hormonal (Teste de comparação entre duas proporções: $p<0,05)$, tinha sido submetida à cirurgia prévia de mama (Teste de comparação entre duas proporções: $p<0,05)$, e encontrava-se com estadiamento III e IV para a neoplasia (Teste de comparação entre duas proporções: $p<0,05)$.

Pelo teste de Qui quadrado, pode-se observar que dados clínicos estudados não tiveram associação estatística ( $p>0,05)$ com 0 afastamento das atividades das pacientes. Para cada variável, a distribuição entre o afastamento e não afastamento do trabalho, tiveram distribuição equivalente (Tabela 2).

A Tabela 3 apresenta a análise descritiva dos efeitos adversos mais descritos na literatura para a população estudada: fadiga, vômito, náusea e insônia.

Não houve presença significativa dos efeitos adversos estudados para o total das pacientes. Porém, quando o grupo estudado foi estratificado pelo afastamento ou não das atividades, a presença de fadiga e náuseas foram estatisticamente significativas nas pacientes afastadas do trabalho (Teste de Qui quadrado: $p<0,05$ ).

Pode-se observar pelo teste $U$ de Mann-Whitney que não houve diferença significativa entre as idades dos dois grupos. Sendo para as afastadas 57 anos $(d p=13,8)$ e para as não afastadas a média foi 55 anos $(d p=14,0)$.

Em relação ao desempenho profissional e doméstico, observou-se, pelo teste $U$ de Mann-Whitney, uma diferença estatisticamente significativa $(p=0,04)$ entre os grupos. 0 grupo que se afastou das atividades apresentou média de incapacidade de realização das atividades diárias maior $(67,0 ; d p=5,6)$ do que o grupo que não se afastou $(49,2 ; d p=6,9)$.

\section{Discussão}

O estudo avaliou dados biodemográficos, clínicos, efeitos adversos do tratamento, relação com a produtividade e indiretamente a qualidade de vida de mulheres com câncer de mama, em tratamento quimioterápico. As pacientes foram estratificadas de acordo com o afastamento ou não das atividades. Quando comparados os parâmetros biodemográficos, a etnia branca foi mais prevalente no grupo não afastadas. As variáveis: estado civil, escolaridade e profissão não tiveram diferença, demonstrando a homogeneidade entre os grupos.

Os dados clínicos: menopausa, reposição hormonal, cirurgia de mama, hipertensão arterial, Diabetes mellitus,

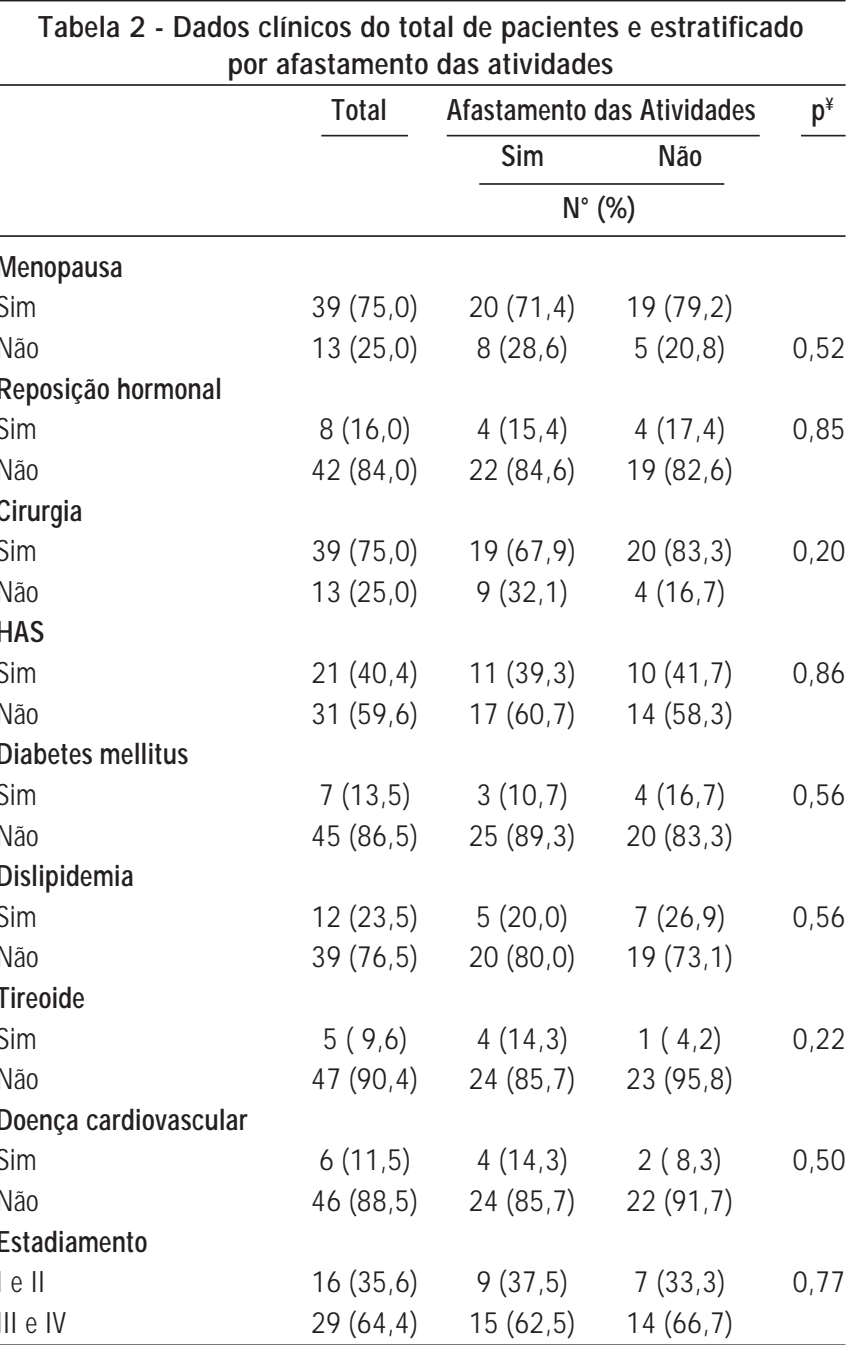

$¥$ : Teste de Qui quadrado entre afastamento ou não das atividades

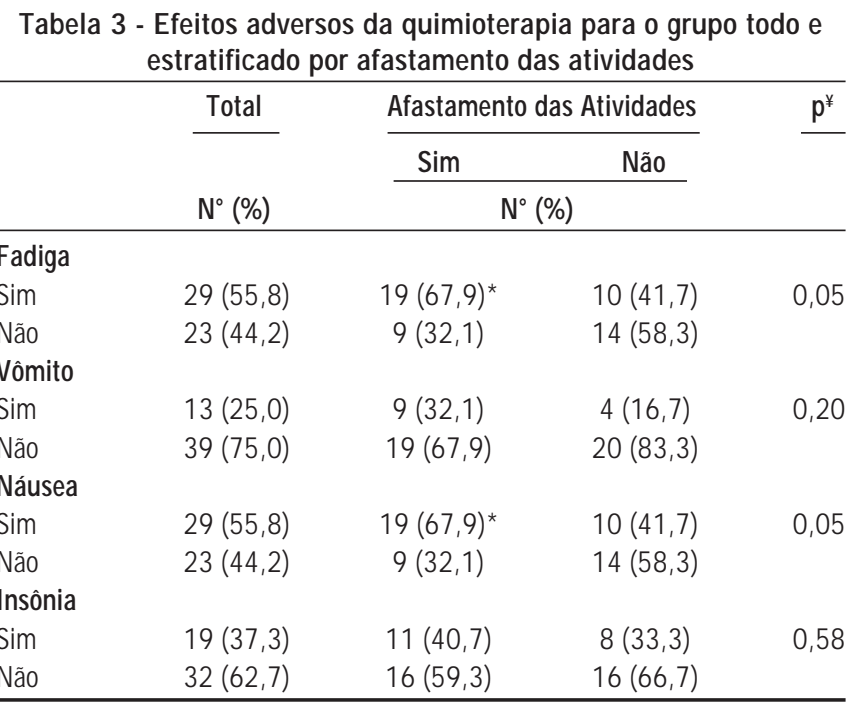

$¥$ : Teste de Qui quadrado entre afastamento ou não das atividades, $* p<0,05$ 
dislipidemia, disfunção da tireóide, doença cardiovascular e estadiamento da neoplasia não foram diferentes entre os grupos.

Embora alguns estudos relatem que 0 tratamento quimioterápico não interfira nas atividades cotidianas ${ }^{18,19}$, observou-se, no presente estudo, o afastamento de quase a metade das pacientes entrevistadas, sendo a maioria com atividade remunerada. Esse fato é de grande relevância, considerando que essas mulheres não só deixaram de contribuir com a renda familiar, como também representaram um custo socioeconômico significativo para o país.

Não há evidências científicas que mudanças na dieta e exercícios físicos melhorem o desempenho físico e psicológico de pacientes com câncer ${ }^{20}$.

Entretanto, a permanência no trabalho auxilia o tratamento, recuperação e o processo da cura $^{12}$. No presente estudo, a maioria das pacientes necessitaram se afastar das atividades em conseqüência do tratamento, e mesmo as mulheres que não se afastaram das atividades, apresentaram alguma incapacidade para a realização das suas atividades durante o tratamento.

Esforços para que permaneçam empregadas, ou em último caso se aposentem em virtude das debilidades ocasionadas pela doença são de extrema importância ${ }^{21}$.

As mulheres que se afastaram de suas atividades apresentaram estadio mais avançado, demonstrando que quanto mais avançada a doença, mais difícil se torna conciliar trabalho e tratamento, provavelmente pela maior agressividade e/ou duração da quimioterapia.

Os sintomas físicos e psicológicos como a fadiga, dor, distúrbios do sono, medo da recorrência da doença, têm sido descritos por mulheres durante e após o tratamento ${ }^{22}$.

Os sintomas fadiga e náuseas, citados pelas pacientes entrevistadas, foram mais prevalentes no grupo das pacientes que se afastaram das atividades, e, de acordo com a literatura, são fatores limitantes importantes para as atividades diárias ${ }^{23}$.

\section{Conclusão}

A quimioterapia acarreta diminuição da capacidade produtiva de parte significativa das mulheres com câncer de mama. 0 estadiamento mais avançado da neoplasia associou-se positivamente ao afastamento das atividades diárias das pacientes, provavelmente decorrente dos efeitos colaterais provocados, como a fadiga e náuseas.

\section{Conflito de interesse: não há}

\section{SUMmARY}

Professional or household performance of patients With BREAST CANCER UNDERGOING CHEMOTHERAPY

OвJECTIVES. Evaluate patients with breast cancer undergoing chemotherapy with respect to their epidemiologic and clinical variables as well as performance at work or at home. METHODS: this was a cross-sectional study including 52 women interviewed during breast cancer chemotherapy, stratified in two groups: those who continued to work and do household tasks, and did not continue to work or do household tasks. Patients were from two public hospitals in the State of
São Paulo, one in Santo Andre and the other in São Bernardo do Campo. The WPAI - GH (Work Productivity and Activity Impairment) questionnaire was used to evaluate work and household performance of professionals or housewives, respectively.

RESULTS. Mean age of the patients was 55.7 ( $S D=13.8)$, most were Caucasian (88.5\%), married (55.8\%), employed $(65.3 \%)$ and the majority had to stop working because of treatment $(51.0 \%)$, at more advanced stage $(p<0.05)$, fatigue and nausea $(p<0.05)$. Mean WPAI - GH was $67.04(\mid S D=$ 5.62 ) for patients who stopped working and 49.17 (SD = 6.89) for those who continued to work (Mann-Whitney $U$ test: $\mathrm{p}=0.04)$.

Conclusion. Chemotherapy leads to a decrease in performance of a sizable fraction of women with breast cancer undergoing chemotherapy. A more advanced stage of neoplasia was positively associated with withdrawal from these activities probably due to side effects such as fatigue and nausea. [Rev Assoc Med Bras 2009; 55(2): 158-62]

KeY Words: Breast neoplasms. Breast. Chemoterapy. Employee performance.

\section{REFERÊNCIAS}

1. Instituto Nacional do Câncer. INCA. [citado 15 julho 2007]. Disponível em:http://www.inca.org.br.

2. Gligio AD. Câncer uma introdução ao seu estudo e tratamento. São Paulo: Pioneira; 1999. v. 1.

3. Pollock RE, Doroshow JH, Khayat D, Nakao A, O’Sullivan B. Manual de oncologia clínica da UICC. União Internacional Contra Câncer. $8^{a}$ ed. São Paulo. Fundação Oncocentro de São Paulo; 2006.

4. Cantinelli FS, Camacho RS, Smaletz O, Gonsales BK, Braguittoni E, Rennó J r J. A oncopsiquiatria no câncer de mama: considerações a respeito de questões do feminino. Rev Psiq Clín. 2006;33(3):124-33.

5. Eifel P, Axelson JA, Costa J. National Institutes of Health Consensus Development Conference Statement: adjuvant therapy for breast cancer, November 1-3, 2000. J Natl Cancer Inst. 2001;93(8):979-89.

6. Curt GA, Breithant W, Cella D, Groopman JE, Horning SJ, Itri LM, et al. Impact of cancer-related fatigue on the lives of patients: new finding from the fatigue coalition. Oncologist. 2000;5(3):353-60.

7. Manocchia M, Reller S, Ware JE. Sleep problems, health- related quality of life, work functioning and health core utilization among the cronicolly ill. Qual Life Res. 2001;10(3):331-45.

8. Gonçalves M; Gíglio J; Ferraz MPT. Depression patients after breast câncer diagnostic/ RBM Rev Bras Med. 2003;60(11):858-59.

9. Spelten ER, Verbeek J H, Uitterhoeve AL, Ansink AC, Van de Lelie J , De Reijke $\mathrm{TM}$, et al. Cancer, fatigue and the return of patients to work: a prospective cohort study. Eur J Cancer. 2003;39(11):1562-7

10. Iconomou G, Mega $V$ Koutras A. Prospective assessment of emotional distress, cognitive function, and quality of life in patients with cancer treated with chemotherapy. Cancer. 2004;101(2):404-11.

11. Gudbergsson SB, Fossa SD, Dahl AA. A study of work changes due to câncer in tumor-free primary-treated câncer patients. A NOCWO study. Support care Cancer. 2008;16(10):1163-71

12. Pettet JR. Cancer and the meaning of work. Gen Hosp Psychiatry. 2000;22(3):200-5.

13. Almeida AM, Prado MAS, Guidorizzi LLF, Rossini FP. Mulheres com câncer de mama: um estudo de morbidade. Acta Oncol Bras. 2002;22(2):263-9.

14. Ciconelli RM, Soárez PC, Kowalski CCG, Ferraz MB. The Brazilian Portuguese Version of the Work Productivity and Activity Impairment General Health (WPAI-GH) Questionnaire. São Paulo Med J. 2006;124(6):325-32.

15. Morettin P, Bussab W. Estatística básica. São Paulo: Atual ED; 1982.

16. Siegel S. Estatística não paramétrica. São Paulo: McGraw-Hill do Brasil; 1981.

17. Kleinbaum DG, Kupper LL, Muller KE, Nizam A. Applied regression analysis and other multivariable methods. Belmont: Duxbury Press; 1998.

18. Hoffman B. Cancer survivors at work: job problems and illegal discrimination. Oncol Nurs Fórum. 1989;16(1):39-43. 
MARTINS LC ET AL.

19. Hoffman B. Employment discrimination: another hurdle for câncer survivors. Cancer Invest. 1991:9(5):589-95

20. Thomas R, Davies N. Lifestyle during and after câncer treatment. Clin Oncol. 2007;19(8):616-27.

21. Mellette SJ. The cancer patient at work. CancerJ Clin. 1985;35(6):360-73.

22. Cappiello M, Cunningham RS, Knobf MT. Breast cancer survivors: information and support after treatment. Clin Nurs Res. 2007;16(4):278-93.
23. Gronvold M, J ensen AB. Patients' experience of breast câncer treatment. U geskr Laeger. 2007;169(37):3114-7.

Artigo recebido: 15/01/08

Aceito para publicação: 27/05/08 